○ Critical Ultrasound Journal a SpringerOpen Journal

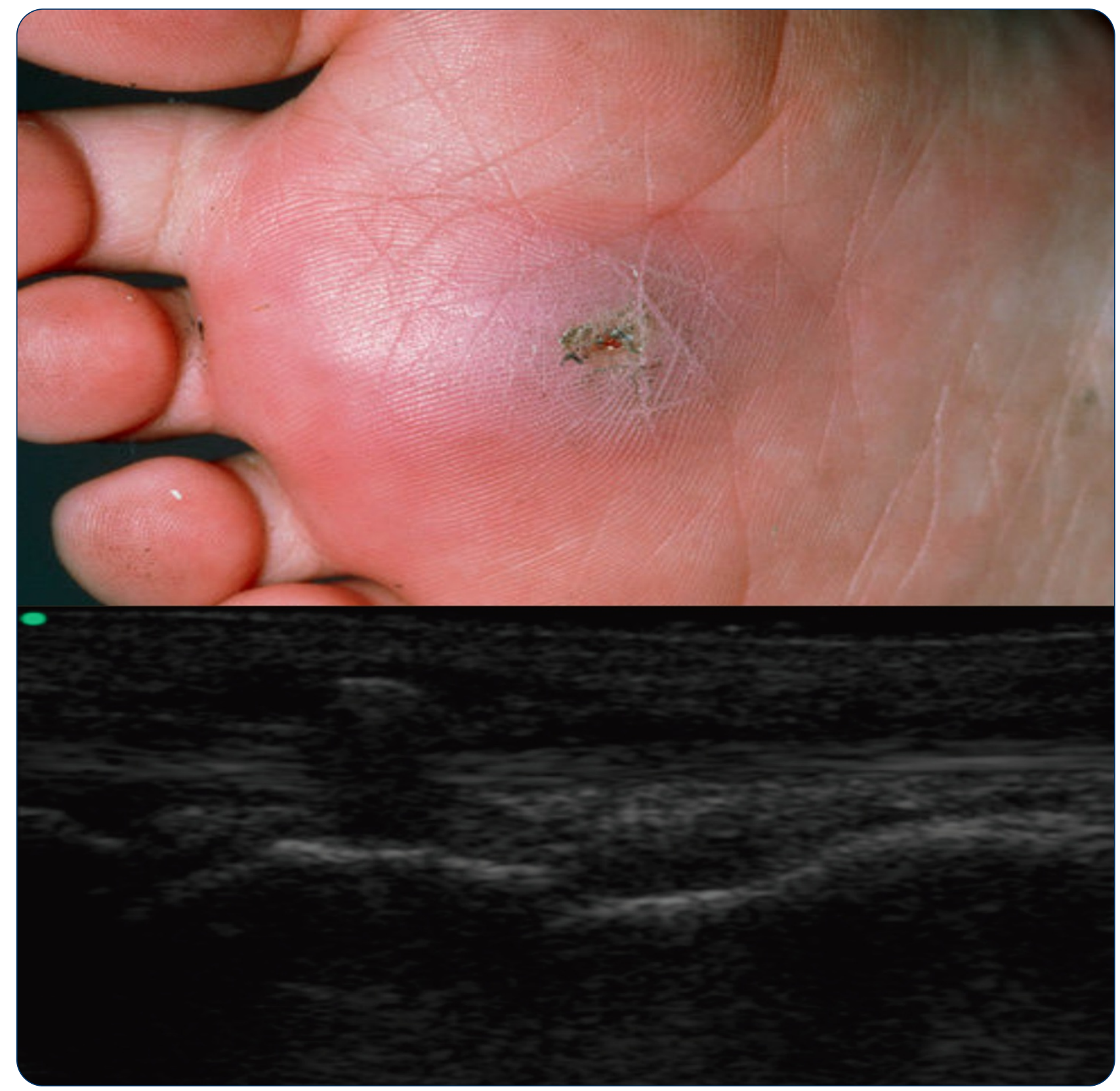

Detection of soft tissue foreign bodies by nurse practitioner-performed ultrasound

Atkinson et al. 


\title{
Detection of soft tissue foreign bodies by nurse practitioner-performed ultrasound
}

Paul Atkinson ${ }^{1,2,3^{*}}$, Rajeev Madan ${ }^{4}$, Richard Kendall ${ }^{4}$, Jacqueline Fraser ${ }^{1}$ and David Lewis ${ }^{1,2}$

\begin{abstract}
Background: This study aimed to evaluate the accuracy of emergency nurse practitioner (NP)-performed point-ofcare ultrasound (POCUS) for the detection of soft tissue foreign bodies (FBs).

Methods: Following a 2-h training session, ten NPs were assessed on their ability to detect various FBs in an experimental model. FBs (wood, metal and plastic) were inserted randomly into eight experimental models (uncooked chicken thighs) by an independent observer. Control experimental models had no FB inserted, but all had a 1-cm incision made on their surface. NPs, blinded to the type of model, were then assessed on their ability to detect the FBs by ultrasound examination using high-frequency linear transducers (Toshiba Nemio). Models were also scanned by two experienced emergency physicians (EPs) as a further control.

Results: Overall, NP-performed POCUS detected 47 of the 60 foreign bodies with a sensitivity, specificity, positive predictive value and negative predictive value of $78.3 \%, 50 \%, 82 \%$ and $43 \%$, respectively, compared with $83.3 \%$, $75 \%, 90.9 \%$ and $60 \%$ for EPs. Sensitivity for detecting specific types of FB was $95 \%, 85 \%$ and $50 \%$ for wood, metal and plastic, respectively, for NP-performed POCUS, compared with 100\%, 100\% and 50\% in the EP group.

Conclusions: NPs with no previous ultrasound experience can detect soft tissue FBs with accuracy comparable to that of EPs in an experimental model. Test sensitivity was high for wood and metal foreign bodies. Specificity was generally low.
\end{abstract}

Keywords: Nurse practitioners; Point-of-care ultrasound; Wound care; Foreign bodies; Diagnosis

\section{Background}

Point-of-care ultrasound (POCUS) is widely used by emergency physicians and radiologists for the detection of soft tissue foreign bodies (FBs) [1].

Previous studies have demonstrated that POCUS has reasonable accuracy for detection of soft tissue FBs, with a range of sensitivities of $69 \%$ to $88 \%$ and specificities of $59 \%$ to $90 \%$ for soft tissue foreign bodies [2-4], and that there is a similar accuracy between different types of sonographers, with sensitivities of $83 \%, 85 \%$ and $74 \%$ and specificities of $83 \%, 85 \%$ and $87 \%$ for radiologists, ultrasound technicians and emergency physicians, respectively [2], and between emergency physicians with a

\footnotetext{
* Correspondence: paul.atkinson@mun.ca

'Emergency Medicine, Saint John Regional Hospital, Saint John, NB E2L 4L4, Canada

${ }^{2}$ Emergency Medicine, Dalhousie University, Halifax, NS B3H 4R2, Canada

Full list of author information is available at the end of the article
}

sensitivity of $97 \%$ and a specificity of $70 \%$ and trainees with a sensitivity of $86 \%$ and a specificity of $83 \%$ [3].

The accuracy of POCUS to detect FBs differs for various substances with one study showing sensitivities for wood of $93 \%$ and plastic of $73 \%$ [4].

Nurses and nurse practitioners are increasingly using POCUS for focused assessment and procedures in the emergency department setting. There is growing evidence that with appropriate training, nursing staff can use POCUS safely $[5,6]$.

Much of the literature supporting nurse- and nurse practitioner-performed POCUS is in the setting of traditional core applications of emergency POCUS such as focused assessment by sonography in trauma (FAST) scanning and ultrasound-guided vascular access [6]. Many nurses and nurse practitioners practice more independently in the minor injury setting in urgent care centres

\section{实}

(c) 2014 Atkinson et al.; licensee Springer. This is an Open Access article distributed under the terms of the Creative Commons Attribution License (http://creativecommons.org/licenses/by/2.0), which permits unrestricted use, distribution, and reproduction in any medium, provided the original work is properly cited. 
and emergency department fast tracks or rapid assessment zones, where they perform initial wound care. Undetected soft tissue FBs can cause delayed wound healing and infection [7]. This study aimed to evaluate the accuracy of emergency nurse practitioner (NP)-performed POCUS for the detection of soft tissue foreign bodies.

\section{Methods}

Study design

The study design was a prospective blinded experimental diagnostic test study. The study was reviewed by the chair of the local research ethics committee, and need for formal approval was waived.

\section{Study setting and population}

The training course and experimental testing were performed at a major tertiary care teaching hospital. Faculty and supervisors were fully accredited by the College of Emergency Medicine in Emergency Point of Care Ultrasound, holding the Certificate in Focused Emergency Ultrasound (CFEU). The nurse practitioners were also staff at the same facility, having achieved full nurse practitioner status and were all actively clinically in the Emergency Department with an annual census of over 90,000 visits per year.

\section{Study protocol}

Ten nurse practitioners each underwent a 2-h hands-on training session by a certified POCUS instructor. This training session consisted of pre-course reading material, a short didactic component (covering the basics of ultrasound physics, instrumentation and the use of ultrasound for detection of soft tissue foreign bodies), and supervised bedside teaching using volunteer models and experimental models (described below). Both the normal ultrasound anatomy of the experimental model and the ultrasound appearance of the various foreign bodies were demonstrated.

In a separate session, following initial training, each NP was individually assessed on their ability to detect various FBs which had been pre-inserted into in an experimental model. The experimental models consisted of wrapped (cling wrap) uncooked chicken thighs each containing either a single metal, glass or plastic FB or no FB (Figure 1). All models were numbered for identification.

Wood, metal and plastic FBs were inserted randomly by an independent observer (also a trained POCUS instructor) into the muscle layer of eight experimental models at a depth of 10 to $15 \mathrm{~mm}$. FBs measured 15 to $20 \mathrm{~mm}$ in length and 1 to $3 \mathrm{~mm}$ in diameter. Control experimental models had no FB inserted. The independent observer recorded which materials were present in each model. All models had a 1-cm incision made on

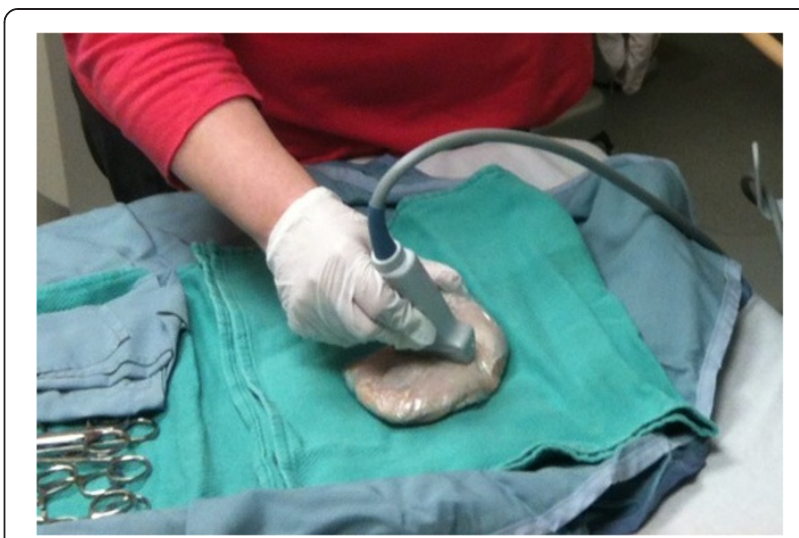

Figure 1 Experimental model consisting of plastic-wrapped chicken thighs containing FBs or none, with a $1-\mathrm{cm}$ incision.

their surface. NPs, blinded to the type of model, were then assessed on their ability to detect the FBs by ultrasound examination (Figure 2) using high-frequency linear transducers (Toshiba Nemio, Toshiba Medical Systems, Tokyo, Japan). For each model, they were asked to record whether an FB was present or absent.

All experimental models were also scanned by two experienced emergency physicians (EPs) as a further quality control measure. These EPs were blinded to the model contents in the same fashion as the NPs. Assessment of accuracy was performed by a third unblinded EP.

Statistical analyses of test characteristics were performed using MedCalc for Windows, version 12.5 (MedCalc Software, Ostend, Belgium).

\section{Results}

Nurse practitioners performed a total of 80 scans, and emergency physicians performed 16 scans (see Table 1). NP-performed POCUS had a sensitivity of $0.783(95 \%$

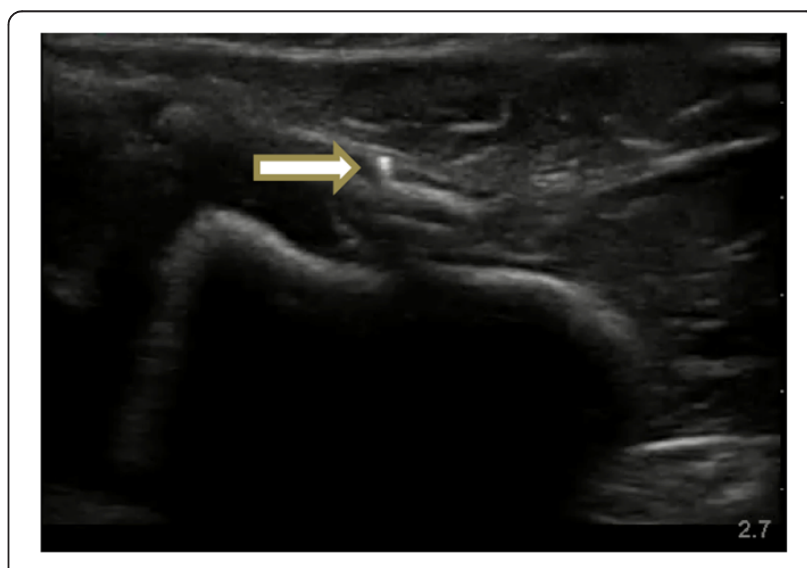

Figure 2 Metal foreign body (arrow) as seen by ultrasound. 
Table 1 Summary of diagnostic test findings for NP- and EP-performed ultrasound for detection of soft tissue FBs

\begin{tabular}{lccc}
\hline & Disease positive & Disease negative & Totals \\
\hline NP & 47 & 10 & 57 \\
Test positive & 13 & 10 & 23 \\
Test negative & 60 & 20 & 80 \\
Totals & & & \\
EP & 10 & 1 & 11 \\
Test positive & 2 & 3 & 5 \\
Test negative & 12 & 4 & 16 \\
Totals &
\end{tabular}

confidence interval (CI) 0.664 to 0.869 ) with a specificity of 0.50 (95\% CI 0.299 to 0.701 ) for detection of soft tissue foreign bodies in the experimental models. EPperformed POCUS had a sensitivity of 0.833 (95\% CI 0.552 to 0.953 ) with a specificity of 0.75 (95\% CI 0.301 to 0.954$)$. Further descriptive diagnostic test statistics for NP- and EP-performed POCUS are shown in Table 2. The sensitivity of both NP- and EP-performed POCUS for different types of foreign bodies was also calculated. NP-performed POCUS had sensitivities of 95\%, 85\% and $50 \%$ for wood, metal, and plastic foreign bodies, respectively. EP-performed POCUS had sensitivities of 100\%, $100 \%$ and $50 \%$ for wood, metal, and plastic foreign bodies, respectively.

\section{Discussion}

This experimental study describes the diagnostic accuracy of nurse practitioner-performed POCUS for the detection of FBs in an experimental model. Using a small group of experienced emergency physicians as an experimental validity control measure, we have demonstrated that following a relatively short and focused period of training, nurse practitioners demonstrated an ability to perform POCUS screening for imbedded soft tissue foreign bodies with reasonable levels of sensitivity. In this experimental model, the sensitivity $(0.783$; $95 \%$ CI 0.664 to 0.869$)$ and specificity $(0.50 ; 95 \%$ CI 0.299 to 0.701$)$ of

Table 2 Descriptive statistics for NP- and EP-performed ultrasound as a test for detection of soft tissue FBs

\begin{tabular}{lccccc}
\hline & \multicolumn{2}{c}{$\mathbf{N P}$} & & \multicolumn{2}{c}{ EP } \\
\cline { 2 - 3 } \cline { 6 - 6 } & Estimate & $\mathbf{9 5 \%} \mathbf{C l}$ & & Estimate & $\mathbf{9 5 \% ~ C l}$ \\
\hline Sensitivity & 0.783 & 0.664 to 0.869 & & 0.833 & 0.552 to 0.953 \\
Specificity & 0.5 & 0.299 to 0.701 & & 0.75 & 0.301 to 0.954 \\
PPV & 0.825 & 0.706 to 0.902 & & 0.909 & 0.623 to 0.984 \\
NPV & 0.435 & 0.256 to 0.632 & & 0.6 & 0.231 to 0.882 \\
LR+ & 1.567 & 0.991 to 2.477 & & 3.333 & 0.599 to 18.543 \\
LR- & 0.433 & 0.226 to 0.831 & & 0.222 & 0.056 to 0.889 \\
\hline
\end{tabular}

NP-performed POCUS compare favourably with historical controls (sensitivity range of 0.69 to 0.88 and specificity range of 0.59 to 0.90 ). It is also apparent that some types of FB (wood and metal) are more easily detected than others (plastic).

\section{Limitations}

It is difficult to conclude that the study shows clinical competence, as the findings were limited to experimental chicken models with artificially inserted foreign bodies. This however is the technique used to train emergency physicians in several accredited courses $[8,9]$.

Other limitations include the time limitation of both the training and testing. The authors' personal experience indicates that the time taken to identify FBs on PoCUS is not uniform and may require prolonged scanning time. Also, this study has low participant numbers, especially on the emergency physician arm, leading to an unbalanced 'control'. This control arm was used as a quality control measure, rather than a true comparative control arm.

\section{Conclusions}

With relatively short focused training, NPs with no previous ultrasound experience can detect soft tissue FBs with accuracy comparable to that of historical controls and EPs in an experimental model.

Test sensitivity was high for wood and metal foreign bodies. Specificity was generally low.

NP-performed POCUS for detection of foreign bodies needs to be evaluated further in a clinical setting before recommending this as an extension of practice.

\section{Competing interests}

All authors have received honoraria for teaching on Emergency Critical Care Ultrasound (ECCUCourse.com) educational ultrasound courses in the UK and Canada.

\section{Authors' contributions}

PA, RM and RK designed the protocol, implemented the experimental model teaching and testing, performed the literature review, drafted the manuscript and reviewed the manuscript. JF and DL reviewed the protocol, contributed to the literature review, drafted the manuscript and reviewed the manuscript. All authors read and approved the final manuscript.

\section{Acknowledgements}

We would like to thank the nurse practitioners at Addenbrooke's Hospital who volunteered as participants in this study.

\section{Author details}

'Emergency Medicine, Saint John Regional Hospital, Saint John, NB E2L 4L4, Canada. ${ }^{2}$ Emergency Medicine, Dalhousie University, Halifax, NS B3H 4R2, Canada. ${ }^{3}$ Emergency Medicine, Memorial University, St John's, NF A1B 3X9, Canada. ${ }^{4}$ Emergency Medicine, Cambridge University Teaching Hospitals, Cambridge, Cambridgeshire CB2 OQQ, UK.

Received: 17 September 2013 Accepted: 13 December 2013 Published: 29 January 2014 


\section{References}

1. Noble VE, Nelson BP (2001) Manual of emergency and critical care ultrasound, 2nd edn. Cambridge University Press, Cambridge

2. Orlinsky M, Knittel P, Feit T, Chan L, Mandavia D (2000) The comparative accuracy of radiolucent foreign body detection using ultrasonography. Am J Emerg Med 18(4):401-403

3. Nienaber A, Harvey M, Cave G (2010) Accuracy of bedside ultrasound for the detection of soft tissue foreign bodies by emergency doctors. Emerg Med Australas 22(1):30-34

4. Hill R, Conron R, Greissinger P, Heller M (1997) Ultrasound for the detection of foreign bodies in human tissue. Ann Emerg Med 29:353-356

5. Blaivas M, Lyon M (2006) The effect of ultrasound guidance on the perceived difficulty of emergency nurse-obtained peripheral IV access. J EMerg Med 31(4):407-410

6. Henderson SO, Ahern T, Williams D, Mailhot T, Mandavia D (2010) Emergency department ultrasound by nurse practitioners. J Am Acad Nurse Pract 22:352-355

7. Imoisili MA, Bonwit AM, Bulas DI (2004) Toothpick puncture injuries of the foot in children. Pediatr Infect Dis J 23(1):80-82

8. Emergency Ultrasound UK and Canada (2013). http://www. emergencyultrasound.net. Accessed 8 July 2013

9. Ultrasound Village. Australia (2009). http://www.ultrasoundvillage.com/. Accessed 8 July 2013

doi:10.1186/2036-7902-6-2

Cite this article as: Atkinson et al:: Detection of soft tissue foreign

bodies by nurse practitioner-performed ultrasound. Critical Ultrasound Journal 2014 6:2.

\section{Submit your manuscript to a SpringerOpen ${ }^{\circ}$ journal and benefit from:}

- Convenient online submission

- Rigorous peer review

- Immediate publication on acceptance

- Open access: articles freely available online

- High visibility within the field

- Retaining the copyright to your article

Submit your next manuscript at springeropen.com 